\title{
Phantoms for diffusion-weighted imaging and diffusion tensor imaging quality control: A review and new perspectives
}

\author{
Edna Marina de Souza ${ }^{1,2 *}$, Eduardo Tavares Costa ${ }^{1,2}$, Gabriela Castellano $^{3,4}$ \\ ${ }^{1}$ Biomedical Engineering Center, University of Campinas, Campinas, SP, Brazil. \\ ${ }^{2}$ Biomedical Engineering Department, Faculty of Electrical and Computer Engineering, University of Campinas, Campinas, SP, \\ Brazil. \\ ${ }^{3}$ Neurophysics Group, Institute of Physics Gleb Wataghin, University of Campinas, Campinas, SP, Brazil. \\ ${ }^{4}$ Brazilian Institute of Neuroscience and Neurotechnology, Campinas, SP, Brazil.
}

Abstract Introduction: Diffusion-weighted imaging (DWI) and diffusion tensor imaging (DTI) combine magnetic resonance imaging (MRI) techniques and diffusion measures. In DWI, the contrast is defined by microscopic motion of water protons. Nowadays, DWI has become important for early diagnostic of acute stroke. DTI images are calculated from DWI images acquired in at least six directions, which give information of diffusion directionality, making it possible to reconstruct axonal or muscle fiber images. Both techniques have been applied to study body structures in healthy and pathological conditions. Currently, it is known that these images and derived parameters are quite sensitive to factors related to acquisition and processing. Magnetic field inhomogeneity, susceptibility, chemical shift, radiofrequency (RF) interference, eddy currents and low signal-to-noise ratio (SNR) can have a more harmful effect in diffusion data than in T1- or T2-weighted image data. However, even today there are not reference phantoms and guidelines for DWI or DTI quality control (QC). Review: Proposals for construction and use of DWI and DTI QC phantoms can be found in literature. DWI have been evaluated using containers filled by gel or liquid with tissue-like MRI properties, as well as using microfabricated devices. DTI acquisitions also have been checked with these devices or using natural or artificial fiber structures. The head phantom from American College of Radiology (ACR) is also pointed out as an alternative for DTI QC. This article brings a discussion about proposed DWI and DTI phantoms, challenges involved and future perspectives for standardization of DWI and DTI QC.

Keywords Magnetic resonance imaging, Diffusion-weighted imaging, Diffusion tensor imaging, Phantoms, Quality control.

\section{Introduction}

DWI reveals microscopic characteristics of water molecules' diffusion across and between biological tissues (Moseley et al., 1990). In these images, the contrast is based on molecular random motion of water protons. It is known that in biological tissues diffusion can be isotropic (having same magnitude in all directions) or anisotropic (following a specific direction). The DWI acquisition involves the application of diffusion-weighting gradients in pulse sequences, creating a signal attenuation due to microscopic water protons' motion. Figure 1 shows the

(c) (1) This is an Open Access article distributed under the terms of the Creative Commons Attribution License, which permits unrestricted use, distribution, and reproduction in any medium, provided the original work is properly cited.

How to cite this article: Souza EM, Costa ET, Castellano G. Phantoms for diffusion-weighted imaging and diffusion tensor imaging quality control: a review and new perspectives. Res Biomed Eng. 2017; 33(2):156-165. DOI: 10.1590/2446-4740.07816.

*Corresponding author: Biomedical Engineering Center, University of Campinas, Rua Alexander Fleming, 163, CEP 13083-881, Campinas, SP, Brazil. E-mail: emarina@ceb.unicamp.br

Received: 29 September 2016/ Accepted: 14 June 2017 pulse sequence used for acquisition of these images in the beginning of 90s (Moseley et al., 1990), based on early studies (Stejskal and Tanner, 1965). This is the basis of modern diffusion pulse sequences.

Rectangular diffusion gradients applied before and after the $180^{\circ}$ refocusing pulse have same magnitude and duration. Thus, if all spins are coherently moving around the magnetic field of the scanner, they will have identical phase and there is no signal related to diffusion. On the other side, when water proton diffusion occurs, static and diffusing spins accumulate different phases, leading to a signal attenuation related to diffusion.

In clinical practice, DWI showed its ability to detect acute stroke (when the symptoms begin) and classify it as ischemic or non-ischemic, while other imaging techniques just reveal brain changes hours after the onset (Moseley et al., 1990). From DWI images the Apparent Diffusion Coefficient (ADC) map and DTI images can be obtained. ADC maps are so called because measured values are lower than those observed when free water diffusion occurs (without interference of compartments and barriers found in biological tissues). The ADC 
calculation described in the Equation 1 is based on two DWI (DWI $I_{i}$ and DWI $)$ obtained with same TE and different diffusion-sensitizing factor (b-value, which units are $\mathrm{s} / \mathrm{mm}^{2}$ ). In this case, TE should be long enough to be compatible with the duration of diffusion gradients, being the $\mathrm{T} 2$-weighted image commonly used.

$$
\mathrm{ADC}_{\mathrm{i}}=-\frac{1}{\mathrm{~b}_{\mathrm{DWI}_{\mathrm{i}}}-\mathrm{b}_{\mathrm{DWI}_{0}}} \ln \frac{\mathrm{DWI}_{\mathrm{i}}}{\mathrm{DWI}_{0}}
$$

where the $i$ index refers to the specific diffusion gradient direction, and $\mathrm{b}_{\mathrm{DWI}_{\mathrm{i}}}$ and $\mathrm{b}_{\mathrm{DWI}_{0}}$ are the $\mathrm{b}$-values of $\mathrm{DWI}$ and $\mathrm{DWI}_{0}$, respectively. Normally, $\mathrm{b}_{\mathrm{DWI}_{0}}$ is taken as 0 , being the $\mathrm{DWI}_{0}$ a T2-weighted image. For brain studies, b typically ranges from $600 \mathrm{~s} / \mathrm{mm}^{2}$ to $1000 \mathrm{~s} / \mathrm{mm}^{2}$.

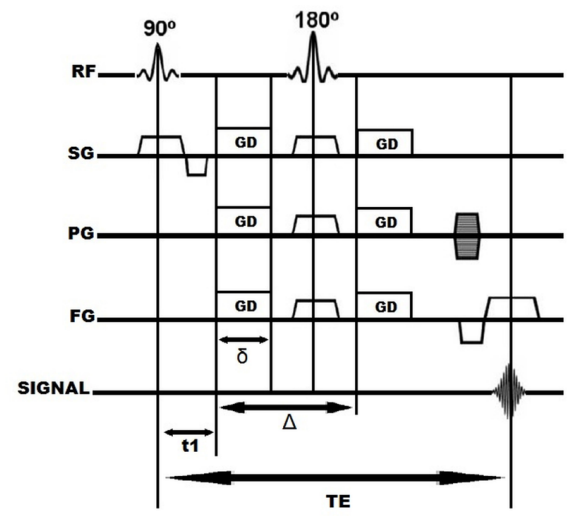

Figure 1. Spin-Echo (SE) pulse sequence for DWI acquisition. RF: radiofrequency; SG: slice selection direction gradient; PG: phase codification direction gradient; FG: frequency codification direction gradient; GD: diffusion-weighting gradient; $\delta$ : duration of GD; $\Delta$ : interval between application of two diffusion-weighting gradients; t1: time from application of RF excitation pulse to the first diffusion-weighting gradient; TE: echo time. The diffusion attenuation of the MRI signal is only dependent on GD, $\Delta$ and $\delta$, but does not depend on $\mathrm{t} 1$.
In diffusion pulse sequences, TE should be long enough to be compatible with the duration of diffusion gradients. Consequently, DWI images are also based on T2 contrast. In terms of contrast, high diffusion areas are typically hypointense and low diffusion areas are hyperintense in DW images (Bammer, 2003). The opposite is seen at ADC maps, as shown in Figure 2.

In the tensor model, principle of DTI, diffusion is described as a multivariate Gaussian distribution and the tensor is a $3 \times 3$ covariance matrix, as shown below:

$T=\left(\begin{array}{ccc}D_{x x} & D_{x y} & D_{x z} \\ D_{y x} & D_{y y} & D_{y z} \\ D_{z x} & D_{z y} & D_{z z}\end{array}\right)$

This matrix describes the displacement of water molecules in three dimensions normalized by diffusion time. The diagonal elements are variances of diffusion along the axes $\mathrm{x}, \mathrm{y}$ and $\mathrm{z}$ and off-diagonal elements are covariance symmetrical terms. Given the tensor symmetry, for its construction DWI images acquired in at least six directions are needed, to obtain the six independent components. To improve the accuracy in tensor estimation it is common to acquire DWI images in 16, 32 or 64 directions. The tensor can also be displayed as an image: and the technique consisting of acquiring DWI images in several directions to build the tensor is called diffusion tensor imaging, or DTI (Basser, 1997). From DTI images, scalar parameters such as fractional anisotropy (FA), relative anisotropy (RA), mean diffusivity (MD) and volume ratio can be extracted. FA is a measure of the fraction of the tensor, which is anisotropic, ranging from 0 (isotropic diffusion) to 1 (completely anisotropic diffusion, i.e., diffusion occurs in a specific direction). RA is the ratio between the anisotropic and isotropic portions of the tensor.

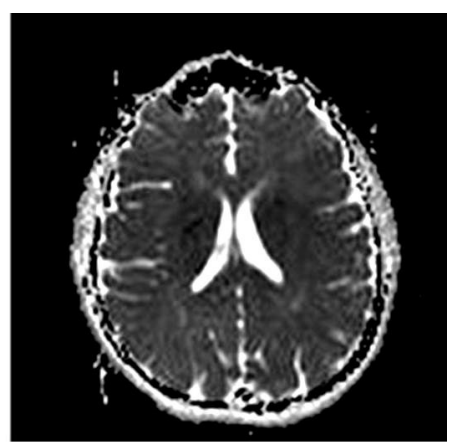

(B)

Figure 2. DWI image and ADC map. Examples of DWI image (A) and ADC map (B) of an adult human brain. Scanner Siemens Verio 3T, acquisition parameters of DWI: TR $/ \mathrm{TE}=5300 / 85 \mathrm{~ms}$, b-values: 0 and $800 \mathrm{~s} / \mathrm{mm}^{2}$. 
MD is the average of the tensor eigenvalues. Finally, volume ratio is a ratio between an ellipsoid volume and a sphere volume, ranging from 1 (isotropic diffusion) and 0 (anisotropic diffusion). DTI data can be used to reconstruct images of axonal fibers (Mori et al., 1999), skeletal muscle (Budzik et al., 2014), and cardiac muscle fibers (Poveda et al., 2013), through tractography techniques. Figure 3 shows an FA image, as well as a color map indicating axonal directions of diffusion.

The reliability of results obtained from DWI and DTI depends on the quality of the acquired data. Low SNR, patient motion during the scan, chemical shift, inhomogeneity of the magnetic field $\mathrm{B}_{0}, \mathrm{RF}$ interference, eddy currents and magnetic susceptibility effects can degrade the quality of DWI and DTI images (Le Bihan et al., 2006). However, there is not a standard phantom or routine for quality control (QC) of these images, as occurs for QC of proton density (PD), T1 and T2-weighted image acquisitions. Development of QC techniques for diffusion images is necessary given the extension of their applications, as well as due to the fact that these images are more sensitive to artifacts when compared to T1, T2 and PD-weighted images, depending on the magnitude and number of short diffusion gradients applied. In other words, changes in the MRI scanner tend to degrade DWI and DTI images before they compromise T1, T2 and PD-weighted scans. Recent studies have suggested phantoms for QC of diffusion acquisitions, based on different kinds of materials and a variety of geometries (Fieremans et al., 2008; Hellerbach et al., 2013; Hubbard et al., 2015; Teh et al., 2016). This work provides an overview of current literature related to this issue, highlighting the progress made and the main challenges to overcome, as well as prospects to standardization of diffusion images' QC.

\section{Methods}

The articles discussed in this work were searched in the data bases PubMed, Web of Science and LILACS. Guidelines of American College of Radiology (ACR), American Association of Physicists in Medicine (AAPM) and of International Society for Magnetic Resonance in Medicine (ISMRM) were also consulted. The searches were done from March 2013 to December 2015. The key-words applied were: diffusion-weighted imaging, diffusion weighted imaging quality control, diffusion tensor imaging, diffusion tensor imaging quality control, magnetic resonance imaging quality control, medical imaging phantoms, MRI phantoms, DWI artifacts, DTI artifacts and MRI instrumentation. The articles were organized according the applications and characteristics of phantom, diffusion pattern simulated (isotropic or anisotropic), as well as the building methods, materials and results.

\section{MRI phantoms described in literature}

The phantoms commonly used in MRI QC routine are not the best option for DWI and DTI images because of their high diffusivity, unlike what occurs in biological tissues. In addition, the compound that fills them is, generally, an aqueous paramagnetic solution containing $\mathrm{CuSO}_{4}, \mathrm{NiCl}_{2}, \mathrm{MnCl}_{2}$ or $\mathrm{GdCl}_{3}$, because of its long-term stability and relaxation time. However, these solutions have T1 very close to T2 (except the $\mathrm{MnCl}_{2}$ ), which does not occur in biological tissues. The results from images of these solutions also depend on the geometry of the container that holds them. Desirable characteristics for a DWI or DTI phantom are covering of a large range of diffusivities, long-term stability, low cost, low toxicity, $\mathrm{T} 1$ and $\mathrm{T} 2$ values similar to biological tissues, and high viscosity in order to minimize vibration and convective motions effects (Kato et al., 2005).

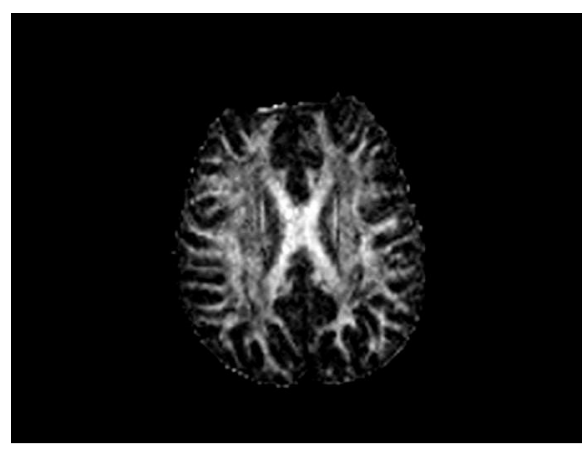

(A)

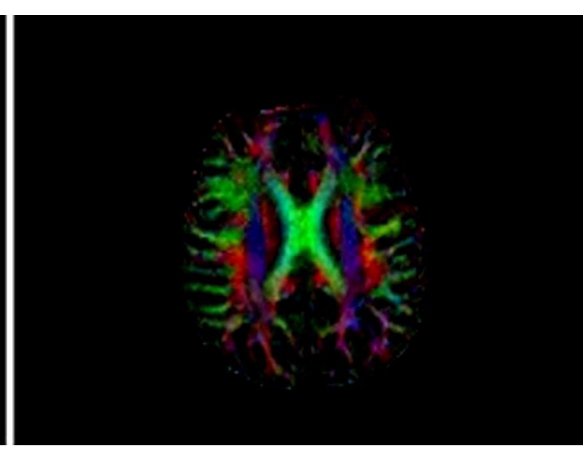

(B)

Figure 3. Images calculated from DTI data of adult brain. (A) FA map; (B) FA color map showing the axonal diffusion directions: red: right-left; green: posterior-anterior; blue: superior-inferior. 
Researchers suggest different kinds of phantoms for DWI and DTI QC, as well as different compounds and solutions to fill them (Madsen and Fullerton, 1982; Pierpaoli et al., 2009; Tofts et al., 2000). There are phantoms of isotropic diffusion for DWI (typically spheres or cylinders filled by liquid) (Laubach et al., 1998; Lavdas et al., 2013; Pierpaoli et al., 2009); fiber phantoms to simulate axonal tracts or cardiac muscle (Fieremans et al., 2008; Lorenz et al., 2008; Teh et al., 2016); phantoms made of capillary or microcapillary arrays permeated by liquid with diffusion properties and/or relaxation times similar to biological tissues (Ebrahimi et al., 2010) test tubes with different solutions (Pierpaoli et al., 2009; Tofts et al., 2000); biological phantoms, such as green asparagus inside a water container (Latt et al., 2007); or animal tissues (axons of pigs or mice) (Chen et al., 2014; Komlosh et al., 2008). There are gels, for isotropic or anisotropic diffusion studies, whose magnetic properties are similar to healthy or pathological tissue (Hellerbach et al., 2013; Kato et al., 2005). Some authors propose applying the ACR MRI head phantom to evaluate scanner parameters related to degradation of DWI and DTI images, such as SNR, low contrast detectability and uniformity (Wang et al., 2011). Computational phantoms can also contribute to QC of DWI and DTI image processing algorithms, being useful to evaluate tractography based on High-Resolution Angle Diffusion Imaging (HARDI), Q-Ball and Diffusion Spectral Imaging (DSI), applicable to analysis of crossing fibers in a voxel (Leemans et al., 2005).

\section{Isotropic diffusion phantoms}

Typically, these phantoms consist of spheres, cylinders or tubes filled by liquids or gels whose relaxation and/or diffusion properties are similar to biological tissues in the MRI environment. A 4.7 T nuclear magnetic resonance (NMR) spectrometer and a 1.5T MRI scanner were used to measure, respectively, self-diffusion coefficients and ADC values of 15 liquid compounds: 3 cyclic alkanes (from cliclohexane to cyclooctane), 9 n-alkanes (from n-octane to n-hexadecane), and 3 alcohols (from ethanol to 1-propanol). The liquids were measured using a standard 5-mm NMR sample tube. n-Tridecane showed ADC values close to normal white matter (WM), in agreement with measures of self-diffusion coefficients obtained from NMR. Nonetheless, despite the advantage of these compounds having defined NMR spectral lines, their use in clinical routine is limited by toxicity and inflammability (Tofts et al., 2000). Non-toxic and stable compounds, such as aqueous solutions of polyvinylpyrrolidone (PVP), can be an alternative to agarose or alkanes. PVP showed long-term stability during a 15 months period (Pierpaoli et al., 2009).
Gels whose dielectric relaxation properties have values close to those found for biological tissue have been tested as filling for isotropic diffusion phantoms. Two gels based on polysaccharide carrageenan (CAG and CAGN) have been explored for isotropic phantoms. CAG consists of carrageenan as a gelling agent, agarose and $\mathrm{GdCl}_{3}$ as $\mathrm{T} 2$ and $\mathrm{T} 1$ modifiers, respectively, in addition to distilled water and the antiseptic $\mathrm{NaN}_{3}$, to reduce gel degradation by microbial agents. The CAGN phantom, besides the compounds already mentioned, also has $\mathrm{NaCl}$ to change electrical conductivity. The concentration of each compound to reach the desirable diffusion properties is defined by an equation set. Those equations can be useful for the development of gels for DWI and DTI QC (Kato et al., 2005). Gels of sodium alginate, xanthan gum, FAVOR-PAC-300, PNC-, Carbomer-980 and Carbopol-974P have also been tested as an alternative to agar or agarose (Hellerbach et al., 2013). It was observed that images of gels had less distortion than images of liquids due to reduction of the macroscopic flow effect. Pathological conditions, such as stroke can also be simulated by gels. A human brain phantom filled by agar-based gel has been applied to analyze DWI pulse sequences performance to discriminate acute stroke and normal gray matter (GM). Changing the gel concentrations of agarose and sucrose is possible to simulate some properties of biological tissues in MRI (T1, T2, ADC-values, for example) such as healthy and pathological brain tissues. With this phantom the researchers observed that $\mathrm{ADC}$ variation between stroke-like areas and GM was similar to that found in human brain (Laubach et al., 1998).

Other kind of container used in DWI phantoms is test tubes. Tubes with different concentrations of a polyacrylamide gel and a sucrose solution were applied to check the performance of Echo-Planar Imaging (EPI) and Half-Fourier Acquisition Single-Shot Turbo Spin-Echo (HASTE) pulse sequences. There were not significant differences between ADC values of both pulse sequences. However, HASTE leads to less ghost and is better to characterize high-value ADC regions (Boursianis et al., 2014). Tubes filled by different concentrations of polyethylene glycol (PEG), to control diffusivity, and Gadobutrol, to control T2, were also tested as isotropic diffusion phantoms (Gatidis et al., 2014). From all studies of isotropic diffusion phantoms, the agar or agarose is the most applied compound. The Table 1 summarizes the characteristics of isotropic phantoms proposed in literature.

\section{Anisotropic diffusion phantoms}

Anisotropic diffusion phantoms are useful for DTI QC.ADTI phantom was made of layers of an agar-based isotropic gel, each layer having a different conductivity. 
Table 1. Isotropic diffusion phantoms found in the literature.

\begin{tabular}{|c|c|c|c|}
\hline Reference & Phantom & Maximum b-value (s/mm²) & $\begin{array}{l}\text { ADC }(\text { Mean } \pm \text { Standard } \\
\text { Deviation })\left(\mathrm{mm}^{2} / \mathrm{s}\right)\end{array}$ \\
\hline Gatidis et al. (2014) & $\begin{array}{l}\text { Tubes filled by PEG and } \\
\text { gadobutrol in different } \\
\text { concentrations }\end{array}$ & 1000 & Ranges: $500,800,1200$ and 2000 \\
\hline Boursianis et al. (2014) & $\begin{array}{l}\text { Test tubes with different } \\
\text { concentrations of } \\
\text { Polyacrylamide gel and } \\
\text { sucrose solution }\end{array}$ & 1000 & Not showed \\
\hline Lavdas et al. (2013) & $\begin{array}{l}\text { Spherical phantom with } \\
\text { compartments filled by gels } \\
\text { (different concentrations of } \\
\text { agarose/sucrose) }\end{array}$ & 1000 & $(1.91 \pm 0.02) \times 10^{-3}$ \\
\hline Kato et al. (2005) & GAG and CAGN compounds & \multicolumn{2}{|c|}{ not calculated } \\
\hline Laubach et al. (1998) & $\begin{array}{l}\text { Brain phantom with } 2 \\
\text { compartments filled by agar } \\
\text { gel and sucrose, to simulate } \\
\text { GM and acute stroke }\end{array}$ & 1000 & $\begin{array}{c}5.5 \times 10^{-4}(\mathrm{ADC} \text { difference of GM } \\
\text { and acute stroke area })\end{array}$ \\
\hline
\end{tabular}

It was shown that gels make the setup more stable and reduce imaging artifacts due to reduction of the macroscopic water flux (Hubbard et al., 2015).

Polycaprolactone (PCL) and polyethylene oxide were used to make axon-like structures using the co-electrospinning technique. The phantom was tested in a preclinical 7T MRI scanner (a scanner dedicated to studies using small animals), and it was found that MD increases when the fiber diameter increases and FA decreases when the fiber internal diameter increases. The setup remained stable for 1 month; however, it was a small phantom, incompatible with clinical scanners (Hubbard et al., 2015). The co-electrospinning technique was also used to develop an anisotropic phantom to simulate cardiac muscle tissue (Teh et al., 2016). Phantoms made using lithography and other microfabrication techniques also been reported to be useful for DTI QC and pulse-sequence tests (Ebrahimi et al., 2010; Komlosh et al., 2011).

Dyneema ${ }^{\circledR}$ fibers are a good option to build DTI phantoms. These fibers have multiple filaments, being more anisotropic than nylon, polyester, polyamide or linen. A phantom consisting of 780 parallel Dyneema ${ }^{\circledR}$ fibers $20 \mathrm{~mm}$ diameter, packed inside a polyolefin tube, was proposed for DTI QC (Fieremans et al., 2008). The FA values of Dyneema ${ }^{\circledR}$ fiber bundles are close to those found in human brain axons and few artifacts are observed on DTI images and tractography. However, the phantom relaxation times differ from those from biological tissues. This may occur because diffusion is limited to the interstitial space between the fibers, where proton density is lower when compared to tubular axons. Despite this, the phantom seems useful to DWI and DTI QC, because their structures are stable and have anisotropy similar to brain tracts (Fieremans et al., 2008).
Parallel and perpendicular Dyneema ${ }^{\circledR}$ fibers were applied to build a multilayer DTI phantom. For DTI images obtained in a 3T scanner, the FA values were also close to those seen in brain WM (Lorenz et al., 2006, 2008).

The stability and reproducibility of DTI parameters have also been checked with organic animal or vegetal phantoms, such as green asparagus inside a water container (Latt et al., 2007), mice nerves (Chen et al., 2014) or pig spinal cord (Komlosh et al., 2008). The Table 2 summarizes characteristics of anisotropic phantoms found in literature.

\section{Discussion}

The development of phantoms and protocols for QC of DWI and DTI images is a subject currently covered by several research groups, given the need for standardization of multicenter/multiscanner studies and also to obtain reliable data for diagnosis in clinical practice. However, there hasn't been agreement about the phantom to be applied or the QC acquisition protocol to detect changes in these images before clinical data degradation. This is due to the wide variety of scanner settings and acquisition techniques. In the reviewed studies, the range of $\mathrm{B}_{0}$ goes from $0.5 \mathrm{~T}$ to $9.4 \mathrm{~T}$. Most studies apply SE-EPI sequences or their clinical/research routines (Lorenz et al., 2006; Teh et al., 2016). New sequences, such as d-PSGE, are being proposed and tested (Komlosh et al., 2008; Lorenz et al., 2008). The d-PSGE sequence was developed for detecting GM microscopic anisotropy, while only macroscopic isotropy is seen with conventional DTI pulse sequences. d-PSGE sequences may be helpful for studies of diseases which degrade GM, such as multiple sclerosis, Alzheimer's disease, Huntington's disease, epilepsy and demyelinating 
Table 2. Anisotropic diffusion phantoms found in the literature.

\begin{tabular}{|c|c|c|c|c|c|}
\hline Reference & Phantom & $\begin{array}{c}\text { Maximum } \\
\text { b-value }\left(\mathrm{s} / \mathrm{mm}^{2}\right)\end{array}$ & $\begin{array}{l}\text { Maximum } \\
\text { NDGD }\end{array}$ & $\begin{array}{l}\text { Maximum MD or } \\
\text { (Mean } \pm \text { Standard } \\
\text { Deviation) } \\
\left(\mathrm{mm}^{2} / \mathbf{s}\right)\end{array}$ & $\begin{array}{c}\text { Maximum FA or } \\
\text { (Mean } \pm \text { Standard } \\
\text { Deviation) }\end{array}$ \\
\hline Kim et al. (2015) & $\begin{array}{l}\text { Capillary array filled by } \\
\text { distilled water and sealed }\end{array}$ & 2000 & 15 & $(1.74 \pm 0.07)$ & $(0.23 \pm 0.005)$ \\
\hline Teh et al. (2016) & $\begin{array}{l}\text { Plastic Syringes with PCL } \\
\text { and PEO }\end{array}$ & 2000 & 30 & $(7.53 \pm 0.16) \times 10^{-4}$ & $(0.388 \pm 0.007)$ \\
\hline $\begin{array}{l}\text { Hubbard et al. } \\
\text { (2015) }\end{array}$ & Fibers of PCL in glass tubes & 800 & 30 & $\begin{array}{l}(3.8 \pm 0.5) \times 10^{-3} \\
(6.1 \pm 0.6) \times 10^{-3}\end{array}$ & $\begin{array}{l}0.45 \pm 0.05 \\
0.33 \pm 0.04\end{array}$ \\
\hline $\begin{array}{l}\text { Hellerbach et al. } \\
\qquad(2013)\end{array}$ & $\begin{array}{l}\text { Polyester fibers around } \\
\text { a cylindrical polyamide } \\
\text { spindle in water }\end{array}$ & 700 & 30 & \multicolumn{2}{|c|}{ Measures of signal intensity } \\
\hline $\begin{array}{l}\text { Lorenz et al. } \\
\qquad(2008)\end{array}$ & $\begin{array}{l}\text { Test of materials to build } \\
\text { fiber phantoms (linen, } \\
\text { viscose, rayon, hemp, } \\
\text { polyamide and Dyneema }{ }^{\circledR} \text {. }\end{array}$ & 1000 & 61 & $\begin{array}{l}(1.4 \pm 0.2) \times 10^{-3} \\
\text { polyamide } \\
(1.1 \pm 0.1) \times 10^{-3} \\
\text { Dyneema }^{\circledR}\end{array}$ & $\begin{array}{l}(0.3 \pm 0.1) \\
\text { polyamide } \\
(0.63 \pm 0.1) \\
\text { Dyneema }^{\circledR}\end{array}$ \\
\hline $\begin{array}{l}\text { Fieremans et al. } \\
\qquad(2008)\end{array}$ & $\begin{array}{l}\text { Dyneema }{ }^{\circledR} \text { fibers tightly } \\
\text { together by a shrinking tube } \\
\text { (in water) }\end{array}$ & 700 & 60 & Not showed & 0.4 \\
\hline $\begin{array}{l}\text { Komlosh et al. } \\
\text { (2008) }\end{array}$ & $\begin{array}{l}\text { Formalin-fixed pig } \\
\text { spinal cord rehydrated } \\
\text { and immersed in } \\
\text { perfluoropolyether }\end{array}$ & 3000 & & Not showed & \\
\hline Latt et al. (2007) & Green asparagus in water & Phan & for test of q & ace diffusion pulse seq & uences. \\
\hline
\end{tabular}

diseases. Out of the brain, these sequences may also be applied to study conditions, which lead to degradation of skeletal, cardiac or smooth muscle (Komlosh et al., 2008).

The studies of DWI and DTI QC tested a variety of setups and materials to build phantoms, although many configurations have received more attention, such as Dyneema ${ }^{\circledR}$ fiber bundles, capillary arrays and containers filled by liquids whose relaxation times are similar to those of biological tissues. QC phantoms for medical imaging, in general, consist of containers filled by liquid to simulate a specific biological tissue or physiological condition being studied. Thinking about water molecules' diffusion, it is possible to simulate their motion both on interstitial and intracellular environments.

Despite the improvement on shimming methods and parallel acquisition techniques, non-homogeneities of $\mathrm{B}_{0}$ lead to geometric distortions in DWI and DTI images. Therefore, in order to build MRI phantoms, it is better to choose containers with well-defined geometry, like spheres or cylinders, which enable identification of imaging distortions without corrupting SNR. Their filling solutions should have relaxation times close to those found for biological tissues. Among some liquids tested, n-tridecane has ADC similar to brain WM. However, cyclic alkanes are toxic and flammable compounds, hindering their use in clinical routine (Tofts et al., 2000). On the other side, sugar-based solutions, despite not being toxic, undergo biological degradation and are not useful for long-term follow-up scanning. The same goes for agarose gels, which are degraded despite the addition of anti-septic agents (Lavdas et al., 2014). PEG and gadobutrol are compounds applied to fill both isotropic and anisotropic phantoms. Alone, PEG has a moderate effect on T2 compared to agarose or sucrose. However, it is efficient to control the range of ADC values. Variation in agarose concentration modifies the $\mathrm{ADC}$ value, while sucrose concentration is related to $\mathrm{T} 2$ values, which can be suitable to simulate biological tissues.

Based on some literature findings, it seems suitable to build a diffusion phantom using both fiber bundles (Fieremans et al., 2008; Lorenz et al., 2008) and compounds like agarose and sucrose (Lavdas et al., 2013). A cylindrical disk may be divided in four compartments with different diffusion patterns. One compartment may have an array of capillaries; other a Dyneema ${ }^{\circledR}$ fiber bundle; the others may be filled by gels containing different concentrations of agarose and sucrose. This disk may be put in a Plexiglas cylinder filled by PEG and gadobutrol and sealed. With this phantom is possible to evaluate both isotropic and anisotropic aspects of diffusion. The PEG and gadobutrol concentrations, as well as the dimensions of the structures applied must be chosen considering the acquisition coil and biological tissue to be simulated. To avoid air bubbles in the phantom a syringe can be used for inserting the solution in the container; remaining bubbles can be 
removed with a vacuum pump. It is important to remove air bubbles to minimize magnetic susceptibility effects. Laundry detergent can favor the elimination of bubbles moving them to the phantom surface, where they can be removed (Lavdas et al., 2014).

It is observed that synthetic polymer-based gels can be a good alternative to agar, because of their long-term stability and easy synthesis (Hellerbach et al., 2013). The main advantage of gel applications in DWI and DTI phantoms (which have fiber bundles and/or other structures where diffusion is anisotropic) is the reduction of flow and convective effects that cause image artifacts (Hellerbach et al., 2013).

Although several studies have compared acquired phantom data with control subject or patient data, human or animal images should never be used as a QC tool for any imaging diagnostic technique. This is because unless body necropsy is done after scanning, the real organization and constitution of organs and tissues are unknown, as well as the patient's physiology. The detection of hardware and software problems through clinical imaging artifacts must be avoided (Van Gemert et al., 2017). Even a specific phantom, when placed at different times in the same equipment, creates distinct $\mathrm{B}_{0}$ distortions. Therefore, images of things whose internal structure and composition are unknown should not be used to find artifact sources, not even to check if a scanner problem was fixed.

The use of a microcapillary-based phantom for DTI QC at the same time of patient imaging seems interesting at first sight, because these structures may have the dimensions of small axons and muscles fibers. However, their use as QC devices shows limitations such as device irreproducibility (their construction is complex) and dependence on microscopy to properly evaluate their structural integrity. Moreover, smaller air bubbles may not be detected and their removal is not easy. In addition, MD and FA values of these phantoms differ significantly from those obtained from biological tissues in most cases, because when phantom structures have dimensions lower than the scanner spatial resolution, bias in MD and FA values may occur (Teh et al., 2016). It is also worth mentioning that the pattern of magnetic field distortion due to the patient's head and to the phantom in the scanner is different from that seen in images of one or the other independently (Kim et al., 2015).

Standard QC MRI phantoms applied to T1, T2 and PD acquisitions, such as ACR phantoms, have diffusivities and relaxation parameters different from biological tissues and are isotropic. They can be helpful to follow parameters such as $\mathrm{B}_{0}$ homogeneity, SNR, presence of ghost and distortions, slice thickness and spatial resolution of those images. However, DWI and DTI are more sensitive to bias compared to conventional
MRI and consequently data corruption occurs first on these images. That is, when a problem is detected on T1, T2 and PD images, probably DWI and DTI are already much degraded.

Generally, one of diffusion studies' goals is the reconstruction of tracts and axonal fibers or even muscle fibers of the body. Thus, ideally, the QC should be applied to devices whose structure and diffusion properties are similar to the ones of those tissues. In other words, the anisotropic portion of the phantom should have MD and FA values closer to those of biological structures. It is known that, in the brain, the average FA for corpus callosum is 0.8 (Mori et al., 1999). For cardiac muscle, in general, it is observed higher MD and lower FA when compared to brain WM (Poveda et al., 2013). To define the materials and compounds to manufacture fiber bundles for DWI/DTI phantoms, some studies showed that Dyneema ${ }^{\circledR}$ fibers, widely applied to engineering, have high anisotropy and are hydrophobic, being useful to simulate brain tracts (Fieremans et al., 2008; Lorenz et al., 2008). Despite the fact of these fiber bundles only allowing interstitial diffusion simulation, they are excellent models for DTI QC when compared to nylon, linen or polyester. However, the anisotropy of bundles depends on fibers' compaction. Higher fiber bundle compression results in higher FA values. The fiber bundles can be compacted by cable ties commonly used in electrical installations. Compressing bundles under water may reduce air bubbles between fibers; a vacuum pump can also help to reduce residual bubbles. Because of the high viscosity of Dyneema ${ }^{\circledR}$, fewer bubbles are formed, minimizing magnetic susceptibility effects. The bundles can be fixed on a Plexiglas container filled by distilled water or PEG and gadobutrol solutions, to control T2 and ADC, respectively. Agar-based gels can also fix bundles in the container and simulate crossing fibers. These gels may minimize scanner vibration effects from the application of magnetic field gradients.

Dyneema ${ }^{\circledR}$ fiber may also simulate cardiac muscle fibers (Teh et al., 2016). In this case, less bundle compression is required when compared to brain tracts' simulations. The improvement of cardiac DTI QC will occur by developing a dynamic cardiac phantom based on flexible microstructures with controlled motions and fiber deformations according to real cardiac physiology. This configuration may be obtained by coupling bundles to a mechanical system connected to an electrocardiogram (ECG) simulator. Thus, it is possible to move the bundles at a specific cardiac frequency and also at irregular frequencies, simulating the health and pathological cardiac physiology at the scan time. To avoid motion artifacts, cardiac gating can be applied (Teh et al., 2016). Thus, a specific phase cardiac cycle is chosen for image acquisition. In general, gating increases the scan time, 
but improves the signal and avoids imaging geometric distortions. For acquisition of patients' data, respiratory and cardiac gating can minimize thorax motion effects.

It is known that different $B_{0}$ intensities and non-homogeneities, as well as pulse sequence parameters, lead to different kinds of $\mathrm{B}_{1}$ distortions. Nonetheless, the main DTI measures (e.g. MD and FA) depend on phantom architecture and composition. Thus, if the phantom has a homogeneous composition, these measures should not vary significantly when changing acquisition protocols and/or $\mathrm{B}_{0}$ intensity, considering images from single or multi-scanner studies.

Regardless of setup and materials applied to DWI and DTI phantoms, some advices can always be considered. First, phantoms filled by water are safer and easier to handle, besides taking a shorter time for thermal equilibrium in the scanner' room. Furthermore, distilled water does not lead to significant signal loss because its T1 and T2 values can match PD dephasing. It is important to watch out for effects of mechanical vibration of the scanner on liquids, which can lead to imaging distortions. For fiber phantoms, whenever possible the bundles should be aligned to $\mathrm{B}_{0}$, avoiding magnetic susceptibility effects. The microscopy and computed tomography (CT) or microCT contribute to the knowledge of internal structures of phantoms, identifying small fissures or misalignments (Chen et al., 2014).

Biological phantoms could be good models for DWI and DTI QC, but they degrade over the time (Chen et al., 2014; Komlosh et al., 2008; Latt et al., 2007). In nature, besides nervous tracts and muscles excised from animals, there are vegetables with highly anisotropic structures such as asparagus, pod and pineapple. Their internal structures can be explored by microCT or microscopy techniques.

Some authors say that is impossible to build a multi-purpose MRI phantom (Hellerbach et al., 2013). They are based on the fact that each technique requires specific structures and materials with $\mathrm{T} 1$ and $\mathrm{T} 2$ compatible to the studied parameter (e.g. SNR, slice thickness, FA and MD from DTI). However, considering the studies here discussed and the commercial phantoms for QC of T1, T2, and PD images, that statement cannot be true. It seems possible to build a single phantom to measure many imaging parameters from PD, T1, T2, DWI and DTI images. For example, a phantom based on an acrylic cylinder whose dimensions fit in the head coil, filled with distilled water. Inside the cylinder, it is possible to position an acrylic disc with each quadrant containing a module for different kinds of MRI QC. A quadrant can support parallel and crossing fibers for DTI QC; a second quadrant may have a relaxation evaluation module; the third and fourth quadrants may have modules for evaluation of isotropic diffusion and CNR, respectively. A quadrant containing PEG and gadobutrol based gels can also be created for DWI evaluation.

In addition to materials and geometries proposed for QC of DWI and DTI, new compounds must be explored or even developed. An example of material that can be useful to build DTI fiber phantoms is the Kevlar ${ }^{\circledR}$ (para-aramid synthetic fiber), a light, strong and durable fiber applied to manufacture of fishing lines, body armor, and bicycle tires, among other applications (Bell et al., 2012). Until now there are no studies applying this material to DTI fiber phantoms' construction. However, there are studies where $\mathrm{Kevlar}^{\circledR}$ is applied to build MRI catheters for performing interventional cardiovascular procedures in the scanner (Bell et al., 2012). The researchers showed that this material does not create imaging distortions from magnetic susceptibility effects, as well as does not reduce SNR.

\section{Conclusion}

DWI and DTI are valuable imaging modalities for medicine, but there is not yet an international standard protocol to check their quality. The studies carried on building a DTI or DWI phantom were not enough to define a standard QC protocol for these images. However, these studies have brought important findings about geometries and compounds to fill the phantoms, as well as how to fabricate their main structures using a variety of techniques. The exploration of these findings should carry on, leading to construction of a standard phantom to evaluate DTI parameters from images acquired with many kinds of MRI scanners.

In conclusion, Dyneema ${ }^{\circledR}$ and synthetic gels with $\mathrm{T} 1, \mathrm{~T} 2$ and $\mathrm{ADC}$ values in the range found in biological tissues are suitable to build anisotropic and isotropic diffusion phantoms, respectively. Furthermore, Dyneema ${ }^{\circledR}$ and gels can be used to build phantoms that evaluate the performance of MRI scanner to measure both isotropic and anisotropic diffusion, as well as can be associated to other structures used in QC of other scanner parameters.

Overall, there is still much work to do about DWI and DTI QC. Besides already known materials promising for this purpose, other ones should be studied or specially developed for the establishment of standard protocols for evaluation of these images.

\section{Acknowledgements}

This work was supported by São Paulo Research Foundation (FAPESP, Brazil - Grant 2013/07559-3) and by the National Council for Scientific and Technological Development (CNPq, Brazil - Grant 310860/2014-8). 


\section{References}

Bammer R. Basic principles of diffusion-weighted imaging. European Journal of Radiology. 2003; 45(3):169-84. PMid:12595101. http://dx.doi.org/10.1016/S0720-048X(02)00303-0.

Basser P. New histological and physiological stains derived from diffusion-tensor MR images. Annals of the New York Academy of Sciences. 1997; 820(1):123-38. PMid:9237452. http://dx.doi.org/10.1111/j.1749-6632.1997.tb46192.x.

Bell JA, Saikus CE, Ratnayaka K, Wu V, Sonmez M, Faranesh AZ, Colyer JH, Lederman RJ, Kocaturk O. A deflectable guiding catheter for real-time MRI-guided interventions. Journal of Magnetic Resonance Imaging. 2012; 35(4):90815. PMid:22128071. http://dx.doi.org/10.1002/jmri.23520.

Boursianis T, Kalaitzakis G, Veneti S, Pappas E, Damilakis J, Maris TG. MRI Diffusion measurements on phantoms: comparison between EPI and HASTE sequences utilizing two fitting methods in Apparent Diffusion Coefficient (ADC) measurements. Physica Medica. 2014; 30:e50. http://dx.doi. org/10.1016/j.ejmp.2014.07.152.

Budzik J-F, Balbi V, Verclytte S, Pansini V, Thuc VL, Cotten A. Diffusion tensor imaging in musculoskeletal disorders. Radiographics. 2014; 34(3):E56-72. PMid:24819802. http:// dx.doi.org/10.1148/rg.343125062.

Chen H, Zhao Y, Zhang T, Zhang H, Kuang H, Li M, Tsien JZ, Liu T. Construct and assess multimodal mouse brain connectomes via joint modeling of multi-scale DTI and neuron tracer data. Med Image Comput Comput Assist Interv. 2014; 17(3):273-80. PMid:25320809.

Ebrahimi B, Swanson SD, Chupp TE. A microfabricated phantom for quantitative MR perfusion measurements: validation of singular value decomposition deconvolution method. IEEE Transactions on Biomedical Engineering. 2010; 57(11):2730-6. PMid:20601306. http://dx.doi.org/10.1109/ TBME.2010.2055866.

Fieremans E, De Deene Y, Delputte S, Ozdemir MS, D’Asseler Y, Vlassenbroeck J, Deblaere K, Achten E, Lemahieu I. Simulation and experimental verification of the diffusion in an anisotropic fiber phantom. Journal of Magnetic Resonance. 2008; 190(2):189-99. PMid:18023218. http://dx.doi.org/10.1016/j. jmr.2007.10.014.

Gatidis S, Schmidt H, Martirosian P, Schwenzer NF. Development of an MRI phantom for diffusion-weighted imaging with independent adjustment of apparent diffusion coefficient values and T2 relaxation times. Magnetic Resonance in Medicine. 2014; 72(2):459-63. PMid:24123316. http://dx.doi. org/10.1002/mrm.24944.

Hellerbach A, Schuster V, Jansen A, Sommer J. MRI phantoms: are there alternatives to agar? PLoS One. 2013; 8(8):e70343. PMid:23940563. http://dx.doi.org/10.1371/journal.pone.0070343.

Hubbard PL, Zhou FL, Eichhorn SJ, Parker GJ. Biomimetic phantom for the validation of diffusion magnetic resonance imaging. Magnetic Resonance in Medicine. 2015; 73(1):299305. PMid:24469863. http://dx.doi.org/10.1002/mrm.25107.

Kato H, Kuroda M, Yoshimura K, Yoshida A, Hanamoto K, Kawasaki S, Shibuya K, Kanazawa S. Composition of MRI phantom equivalent to human tissues. Medical Physics.
2005; 32(10):3199-208. PMid:16279073. http://dx.doi. org/10.1118/1.2047807.

Kim SJ, Choi CG, Kim JK, Yun SC, Jahng GH, Jeong HK, Kim EJ. Effects of MR parameter changes on the quantification of diffusion anisotropy and apparent diffusion coefficient in diffusion tensor imaging: evaluation using a diffusional anisotropic phantom. Korean Journal of Radiology. 2015; 16(2):297-303. PMid:25741191. http://dx.doi.org/10.3348/ kjr.2015.16.2.297.

Komlosh ME, Lizak MJ, Horkay F, Freidlin RZ, Basser PJ. Observation of microscopic diffusion anisotropy in the spinal cord using double-pulsed gradient spin echo MRI. Magnetic Resonance in Medicine. 2008; 59(4):803-9. PMid:18383293. http://dx.doi.org/10.1002/mrm.21528.

Komlosh ME, Ozarslan E, Lizak MJ, Horkay F, Schram V, Shemesh N, Cohen Y, Basser PJ. Pore diameter mapping using double pulsed-field gradient MRI and its validation using a novel glass capillary array phantom. Journal of Magnetic Resonance. 2011; 208(1):128-35. PMid:21084204. http:// dx.doi.org/10.1016/j.jmr.2010.10.014.

Latt J, Nilsson M, Rydhog A, Wirestam R, Stahlberg F, Brockstedt S. Effects of restricted diffusion in a biological phantom: A q-space diffusion MRI study of asparagus stems at a 3T clinical scanner. Magma. 2007; 20(4):213-22. PMid:17952478. http:// dx.doi.org/10.1007/s10334-007-0085-z.

Laubach HJ, Jakob PM, Loevblad KO, Baird AE, Bovo MP, Edelman RR, Warach S. A phantom for diffusion-weighted imaging of acute stroke. Journal of Magnetic Resonance Imaging. 1998; 8(6):1349-54. PMid:9848751. http://dx.doi. org/10.1002/jmri.1880080627.

Lavdas I, Behan KC, Papadaki A, McRobbie DW, Aboagye EO. A phantom for diffusion-weighted MRI (DW-MRI). Journal of Magnetic Resonance Imaging. 2013; 38(1):173-9. PMid:23576443. http://dx.doi.org/10.1002/jmri.23950.

Lavdas I, Miquel ME, McRobbie DW, Aboagye EO. Comparison between diffusion-weighted MRI (DW-MRI) at 1.5 and 3 tesla: a phantom study. Journal of Magnetic Resonance Imaging. 2014; 40(3):682-90. PMid:24925470. http://dx.doi. org/10.1002/jmri.24397.

Le Bihan D, Poupon C, Amadon A, Lethimonnier F. Artifacts and pitfalls in diffusion MRI. Journal of Magnetic Resonance Imaging. 2006; 24(3):478-88. PMid:16897692. http://dx.doi. org/10.1002/jmri.20683.

Leemans A, Sijbers J, Verhoye M, Van der Linden A, Van Dyck D. Mathematical framework for simulating diffusion tensor MR neural fiber bundles. Magnetic Resonance in Medicine. 2005; 53(4):944-53. PMid:15799061. http://dx.doi. org/10.1002/mrm.20418.

Lorenz R, Bellemann ME, Hennig J, Il'yasov KAAnisotropic phantoms for quantitative diffusion tensor imaging and fiber tracking validation. Applied Magnetic Resonance. 2008; 33(4):419. http://dx.doi.org/10.1007/s00723-008-0087-7.

Lorenz R, Kreher BW, Henning J, Bellemann ME, Il'yasov KA. Anisotropic Fiber Phantom for DTI validation on a clinical scanner. In: Proceedings of the 14th International Society for Magnetic Resonance in Medicine (ISMRM); 2006; Seattle, USA. Seattle: ISMRM; 2006. p. 2738. 
Madsen EL, Fullerton GD. Prospective tissue-mimicking materials for use in NMR imaging phantoms. Magnetic Resonance Imaging. 1982; 1(3):135-41. PMid:6927201. http:// dx.doi.org/10.1016/0730-725X(82)90204-1.

Mori S, Crain BJ, Chacko VP, Van Zij1 PC. Three-dimensional tracking of axonal projections in the brain by magnetic resonance imaging. Annals of Neurology. 1999; 45(2):265-9. PMid:9989633. http://dx.doi.org/10.1002/1531-8249(199902)45:2<265::AIDANA21>3.0.CO;2-3.

Moseley ME, Cohen Y, Mintorovitch J, Chileuitt L, Shimizu H, Kucharczyk J, Wendland MF, Weinstein PR. Early detection of regional cerebral ischemia in cats: comparison of diffusion- and T2-weighted MRI and spectroscopy. Magnetic Resonance in Medicine. 1990; 14(2):330-46. PMid:2345513. http://dx.doi. org/10.1002/mrm.1910140218.

Pierpaoli C, Sarlls U, Nevo PJ, Basser P, Horkay F. Polyvinylpyrrolidone (PVP) water solutions as isotropic phantoms for diffusion MRI studies. In: Proceedings of the 17th International Society for Magnetic Resonance in Medicine (ISMRM); 2009; Honolulu, Hawaii, USA. Seattle: ISMRM; 2009. p. 1414.

Poveda F, Gil D, Martí E, Andaluz A, Ballester M, Carreras F. Helical structure of the cardiac ventricular anatomy assessed by diffusion tensor magnetic resonance imaging with multiresolution tractography. Revista Espanola de
Cardiologia. 2013; 66(10):782-90. PMid:24773858. http:// dx.doi.org/10.1016/j.recesp.2013.04.022.

Stejskal EO, Tanner JE. Spin diffusion measurements: spin echoes in the presence of a time-dependent field gradient. The Journal of Chemical Physics. 1965; 42(1):288-92. http:// dx.doi.org/10.1063/1.1695690.

Teh I, Zhou FL, Hubbard Cristinacce PL, Parker GJ, Schneider JE. Biomimetic PHANTOM FOR CARDIAC DIFFUSION MRI. Journal of Magnetic Resonance Imaging. 2016; 43(3):594600. PMid:26213152. http://dx.doi.org/10.1002/jmri.25014.

Tofts PS, Lloyd D, Clark CA, Barker GJ, Parker GJ, McConville P, Baldock C, Pope JM. Test liquids for quantitative MRI measurements of self-diffusion coefficient in vivo. Magnetic Resonance in Medicine. 2000; 43(3):368-74. PMid:10725879. http://dx.doi.org/10.1002/(SICI)1522-2594(200003)43:3<368::AIDMRM8>3.0.CO;2-B.

Van Gemert J, Brink W, Webb A, Remis R. An efficient methodology for the analysis of dielectric shimming materials in magnetic resonance imaging. IEEE Transactions on Medical Imaging. 2017; 36(2):666-73. PMid:27831868. http://dx.doi. org/10.1109/TMI.2016.2624507.

Wang ZJ, Seo Y, Chia JM, Rollins NK. A quality assurance protocol for diffusion tensor imaging using the head phantom from American College of Radiology. Medical Physics. 2011; 38(7):4415-21. PMid:21859042. http://dx.doi. org/10.1118/1.3595111. 